\title{
La observación de las representaciones mediáticas del medio ambiente en la prensa. El caso de La Crónica de Baja California, México
}

The observation of the environment media representations in the press. The case of La Crónica de Baja California, Mexico

\author{
Ángel Manuel ORTIZ MARín \\ Universidad Autónoma de Baja California - México \\ mortiz@uabc.edu.mx
}

\begin{abstract}
Cómo citar este artículo: ORTIZ MARín, Ángel Manuel (2015): "La observación de las representaciones mediáticas del medio ambiente en la prensa. El caso de La Crónica de Baja California, México», Mediaciones Sociales, no 14, pp. 59-79. DOI: http://dx.doi.org/10.5209/rev_MESO.2015.n14.51559
\end{abstract}

Recibido: 17 de septiembre de 2015.

Aceptado: 27 de noviembre de 2015.

\section{RESUMEN}

Observar las representaciones sociales expresadas en los medios de comunicación masiva, en particular en la prensa, ha sido una tarea del Observatorio Global Mediático de la Frontera Norte de la Universidad Autónoma de Baja California (Observa/Frontera) desde hace seis años; en esta ocasión un conjunto de observatorios de medios en México se abocó a la tarea de observar las representaciones mediáticas en torno al tema del medio ambiente y sus diferentes problemáticas en la prensa mexicana. Sustentado en la teoría de la Agenda Setting (McCombs y Shaw, 1972), Observa/Frontera realizó un análisis durante cuatro semanas de las notas periodísticas publicadas en el diario La Crónica de Baja California y los resultados obtenidos de las 34 notas registradas muestran que desafortunadamente los aspectos ambientales o de cambio climático son escasamente abordados y, cuando lo refieren, es por la ocurrencia de catástrofes ya sean naturales o provocadas por el hombre.

PalABRAS ClAVE: Agenda Setting, medio ambiente, observatorio, prensa, representaciones mediáticas. 


\begin{abstract}
Observe the social representations expressed in the media, particularly in the press, has been one of the objective of The Observatorio Global Mediático de la Frontera Norte de la Universidad de Baja California (Observa/Frontera) for the last six years. In this time, a team of media researchers in Mexico took up the task of observing the media representations regarding the environment and its issues in the Mexican press. Based on the Agenda Setting theory (McCombs y Shaw, 1972), Observa/Frontera made an analysis for 4 weeks of all the press articles published in the journal $L a$ Crónica de Baja California. The result obtained after researching the 34 articles published in the mentioned journal, shows that unfortunately it rarely deals with environment or climate change topics and when it happens, it is only attributed to a catastrophic causes made by nature or humans.
\end{abstract}

KEYWORDS: Agenda Setting, environment, observatory, press, media representations.

Sumario: 1. Introducción. 2. La representación mediática de la temática ambiental. 3. Metodología para el análisis ambiental. 4. Objeto de estudio y objetivos de la investigación. 5. Abordaje teórico. 6. Contexto periodístico en Baja California. 7. Metodología y organización de la investigación. 8. Análisis de los resultados. 9. Conclusiones. Bibliografía.

\title{
1. INTRODUCCIÓN
}

La problemática del medio ambiente suscita, en la mayoría de las ocasiones, comentarios favorables, tanto de expertos como de quienes no lo son, en torno a la preservación del medio ambiente. Sin embargo, otros problemas igual de graves y complejos, que aquejan a la humanidad como la pobreza extrema o el hambre, son asuntos de la mayor atención de los organismos nacionales e internacionales.

El 17 de junio del 2015, la directora general de la UNESCO, Irina Bokova, emitió un mensaje con motivo del Día Mundial de la Lucha contra la Desertificación, en el cual hizo mención de que

"La desertificación y la degradación de los suelos suponen una amenaza para la seguridad alimentaria, la estabilidad y la paz, a la que debemos hacer frente con rapidez y determinación y actuando en múltiples frentes. Estos problemas atañen a las comunidades directamente afectadas, pero son importantes para todos nosotros, en todo el mundo" (Unesco, 2015: 2).

El problema no es menor, pues incluso hay un conjunto de organismos internacionales que han integrado una enorme red de colaboración para atender los diversos rasgos del medio ambiente, uno de ellos es el agua, dado que es un problema que se avizora a nivel mundial, como recurso no renovable del cual la humanidad depende para su sobrevivencia. Por tal razón, hay organismos tanto nacionales, internacionales 
y supranacionales que están abocados a plantear alternativas para la preservación del uso social del agua, tanto a nivel doméstico, industrial como agrícola.

El pasado 31 de agosto de este año, se llevó cabo en Anchorage, Alaska, la conferencia Liderazgo Mundial en el Ártico: Cooperación, Innovación, Compromiso y Adaptación con la presencia de representantes de 20 países. En ella, los dignatarios de las naciones participantes firmaron una declaración conjunta sobre el cambio climático, particularmente a partir de las advertencias de científicos que anticipan que las temperaturas del Ártico están provocando severos cambios en el planeta, incluso el presidente Obama al clausurar dicha cumbre climática afirmó que no se está haciendo lo suficiente, al referirse a los compromisos de las naciones por preservar el medio ambiente (Yona, 2015).

Este problema es cercano a México, por las diferentes zonas desérticas con las que cuenta el país, y en particular, la ciudad de Mexicali, Baja California, la cual está enclavada en una de estas zonas que corresponde al Ilamado Gran Desierto de Sonora, en la región noroeste de la república mexicana.

Esta es la razón por la cual, este texto documenta la importancia de cómo se visibiliza en los medios de comunicación, la problemática del medio ambiente en sus diferentes expresiones, como es el clima, el agua y la energía.

\section{LA REPRESENTACIÓN MEDIÁTICA DE LA TEMÁTICA AMBIENTAL}

Las condiciones en las que se documenta la importancia de los sucesos que refieren los medios de comunicación masivos (MCM), tanto impresos, electrónicos y digitales, alude el gran impacto en la agenda pública de los gobiernos, y cuando el tema es el medio ambiente, se podría partir de la premisa de que es una de las preocupaciones fundamentales de las mayoría de las naciones, particularmente las industrializadas y las que no lo son.

Ya Martín Serrano (1985) refiere que los medios operan, según el conflicto por informar, desde la mediación cognitiva, pues al documentar el nuevo acontecer que rompe con la cotidianeidad, aquel compromete la normalidad social, de ahí que los medios de comunicación tienden a ajustar el nuevo relato del hecho a las normas y valores socialmente legitimados.

Esta condición alude indiscutiblemente el relato noticioso de aquellos problemas del medio ambiente, pues cuando ocurren desastres naturales cuya afectación a la vida humana o a la flora y fauna es considerable, el impacto mediático es de proporciones excepcionales por un determinado tiempo, pero la noticia deja de serlo en breve tiempo hasta que los afectados por el percance ambiental vuelven a la "normalidad" de su cotidianeidad. 
De ahí la importancia de que los códigos de representación mediática del mundo se mantengan estables, a pesar de que el contenido del relato de los aconteceres cambie o sea distorsionador de la normalidad (Martín Serrano, 1977).

Por otra parte, los medios de comunicación y los periodistas (con las debidas excepciones), se han convertido en voceros de los grandes transnacionales que han depauperado los recursos naturales, ya sea los bosques, el agua y afectado enormemente el clima, al grado de que el calentamiento global ya es una frase de uso común y a veces, erróneamente utilizada.

Sin embargo, periodísticamente son los deportes, los espectáculos, los sucesos políticos relevantes o escandalosos, los crímenes y las crisis financieras las que ocupan en general las primeras, segundas y terceras páginas de los principales periódicos del mundo. Es la tónica periodística, las noticias malas llaman más la atención y suelen vender más ejemplares. Ciertamente la conciencia sobre los problemas del medio ambiente ya está el vocabulario de la mayor parte de la población. En la escuela, en la familia, en el trabajo y en algunos medios hay referencia a condiciones que aluden a la problemática del medio ambiente y sus diferentes secuelas en las condiciones de vida de los habitantes del planeta.

Pero los medios en general, le prestan atención de manera esporádica, o sólo cuando hay catástrofes que afectan a amplios sectores de la población. Inundaciones, tifones, huracanes, seísmos, incendios de bosques o plagas, suelen ser de interés periodístico por las dimensiones de la afectación a la población y a la naturaleza.

Shanahan (2007) da cuenta de que en Inglaterra, una consultora de comunicación realizó una investigación sobre la publicación de noticias relacionadas con el cambio climático en dos diarios ingleses de la mayor circulación nacional e internacional, como Financial Times (circulación diaria de 450 mil ejemplares) y The Sun (3,1 millones), de agosto a noviembre del 2005, el primero publicó 23 por ciento de noticias relacionadas con el cambio climático y el segundo, con 1,5 por ciento. Lo que puede indicar que en la agenda temática de esos diarios, los problemas suscitados por el cambio climático no tuvieron la relevancia adecuada al nivel de la gravedad del problema que enfrenta cotidianamente la humanidad en este tema.

De ahí, que las representaciones mediáticas sobre el tema del medio ambiente ocurren según la incidencia de determinados factores. Ya sea cuando hay algún evento internacional que congrega a numerosos especialistas sobre el tema del medio ambiente, cambio climático o calentamiento global; cuando algún político carismático alude el tema y/o habla de los esfuerzos de las naciones industrializadas para proteger el medio ambiente, como el caso de Al Gore, o en la ocurrencia de desastres climáticos que conmueven por su impacto en la estabilidad o los destrozos que ocasionan a los seres humanos o a la fauna y flora. 
Estos parecieran momentos en los cuales los aconteceres no pueden ser eludidos, son picos en los que los medios les otorgan atención, pero después hay amplios silencios y omisiones en torno a esfuerzos ya sean gubernamentales, de grupos ambientalistas o de la sociedad organizada por actuar a favor de preservar el medio ambiente.

Garivati (2013) refiere dos modelos de periodismo en torno al relato del acontecer ambiental: el periodismo ambiental empírico y el periodismo ambiental conceptual. Al primero lo cita como el discurso periodístico actual en el cual hay ya una paulatina especialización profesional, modalidad de formación todavía incipiente en México, tanto en las escuelas y facultades de periodismo, como en la práctica profesional. Al otro modelo, el autor lo ubica como desde tres vertientes, "el discurso de los movimientos sociales ambientalistas, desde el discurso político y el propio discurso académico" (Garivati, 2013: 218).

Evidentemente la problemática del medio ambiente, cuyas repercusiones afecta de diferentes maneras, tanto en el clima, el agua, como la energía, suele traer aparejados efectos que provocan desastres en las regiones en los cuales se desencadenan fenómenos que por su naturaleza e impacto ambiental generan pérdidas humanas, materiales o de la flora y la fauna de las zonas en las cuales se manifiestan dichos desastres. Es justamente en esos momento, en los cuales los medios de comunicación masiva, ya sean impresos, electrónicos o digitales, pueden coadyuvar en prevenir, orientar y alerta a la población sobre el desencadenamiento de fenómenos como un huracanes, tsunamis, tornados, deslaves de tierra, sequías, inundaciones o incendios. Otros no son predecibles como los seísmos o aquellos que el hombre provoca consciente e inconscientemente.

Para ello, se requiere de una conciencia de la innovación sostenible como la que refiere Díaz Nosty (2009) al señalar que el binomio comunicación y sostenibilidad implica una comunicación para el desarrollo sostenible, en la cual los medios de comunicación juegan un papel educador y orientador, cuya data proviene del papel difusionista de la escuela norteamericana. Pues bien, la propuesta de este académico de la Universidad de Málaga, la sustenta como "una estrategia que encierra en sí misma una instancia de innovación, ya que plantea un cambio en la conciencia narrativa de los medios y una vertiente propositiva vinculada a su potencial difusión" (Díaz Nosty, 2009: 3).

En México, las políticas públicas sobre medio ambiente han transitado por diferentes posturas oficiales, las más de ellas sólo discursivas y fue hasta 1994 cuando el entonces presidente Ernesto Zedillo creó la Secretaría de Medio Ambiente, Recursos Naturales y Pesca (Semarnap) con el objetivo de proteger y restaurar los ecosistemas y recursos naturales (Borrego, 2006). De ello derivó un año más tarde una consulta que convocó la Comisión de Ecología de la Cámara de Diputados, lo cual estableció un 
importante precedente al abrir a la participación ciudadana la discusión de los temas ambientales. Los resultados no fueron los esperados pues fueron escasas las propuestas que surgieron de dicha iniciativa, como para dar forma a un marco legal que correspondiera a una legislación ambiental acorde a las condiciones ambientales prevalecientes en el mundo.

Según la autora, la participación ciudadana fue escasa también a causa "de la ausencia de información para los ciudadanos por parte de las autoridades ambientales" (Borrego, 2006: 184) aunado a que "los organismos empresariales, rechazaron desde un principio, la ampliación de la participación social en la formulación, gestión y aplicación de la política ambiental" (Borrego, 2006: 185).

Si aunamos a estas condiciones de política gubernamental, la escasa o nula información sobre la preservación del medio ambiente para la sociedad mexicana y en particular a la ciudadanía bajacaliforniana, se puede entender por qué en la agenda de los medios de comunicación masiva no está el tema ambiental. Podría también formularse la hipótesis de que el ocultamiento de esta problemática es determinada por grupos de poder políticos o empresariales que impiden a los medios divulgar información adecuada para desalentar la ampliación de los cauces de participación ciudadana en la demanda de sus derechos ambientales.

Habrá que entender que la condición de masivo a los MCM no proviene de la cantidad de individuos que acceden a los productos comunicativos, sino de las condiciones en que estos están disponibles y su accesibilidad a los consumidores de los mismos. De ahí que Thompson (1997: 45) refiera que el concepto masivo se ha confundido y dado asociaciones que implican a "sujetos pasivos e indiferenciados". De ahí que dicho término sea equivocadamente aplicado en el análisis de las representaciones mediáticas. Dicho autor, indica que las características de la comunicación masiva se relacionan con la producción de bienes simbólicos al conllevar el desarrollo de innovaciones tecnológicas que operan en el escenario mediático. Una segunda condición refiere Thompson (1997) es que este avance tecnológico implica el surgimiento y expansión a su vez de las industrias mediáticas, las cuales impulsan el consumo de formas simbólicas que se reproducen mediante diferentes formas de mensajes o relatos de los aconteceres. Son estos aconteceres o relato de los hechos, los que cobran importancia a partir de ser representados mediáticamente y a su vez, consumidos por los diferentes públicos que acceden a la información noticiosa, como es el caso que trata este texto. Por lo que, las condiciones en las que se conforma la importancia de los sucesos que documentan los MCM, tanto impresos, electrónicos como digitales, refiere el gran impacto en la agenda pública de los gobiernos desde la perspectiva de la Agenda Setting. 
Lo grave de la situación en el tema ambiental, es que nuevamente son los hechos catastróficos los que ocasionan que la atención mediática visibilice aquellas situaciones en las que ocurre un desastre ambiental, como la derrama de petróleo en el mar; el verter químicos en ríos y lagos que se utilizan para el consumo humano o agrícola; la quema de pastizales o el provocar incendios forestales con fines de ampliar espacios para el cultivo agrícola, ante lo cual la ciudadanía no cuenta con las instancias para exigir la reparación del daño.

\section{Metodología PARA EL ANÁLISIS AMBIENTAL}

El 2014, la Red de Observatorios Mediáticos del Consejo Nacional para la Enseñanza y la Investigación de las Ciencias de la Comunicación (ROM-CONEICC), a propuesta del Observatorio Veracruzano de Medios del Centro de Estudios de Opinión y Análisis de la Universidad Veracruzana (OBVIO) invitó a los observatorios adscritos a la ROM a participar de la primera fase de un proyecto de investigación cuyo objetivo fue analizar los temas que publica la prensa impresa en México sobre el tema medio ambiente y sus principales problemas. Para tal fin se determinó de forma homogénea, tanto un modelo teórico-metodológico, que para el caso fue la vertiente de Agenda Setting propuesta por McCombs y Shaw (1972), así como una técnica que fue el análisis de contenido cuantitativo dentro del modelo de Berelson (1952).

En este proceso de análisis participaron seis observatorios de las universidades de: Autónoma de Coahuila, campus Torreón; Autónoma de Coahuila, campus Saltillo; Autónoma de San Luis Potosí, Autónoma de Baja California, el Instituto Tecnológico de Estudios Superiores de Occidente (ITESO); Universidad Veracruzana y un investigador de la Universidad Autónoma de Yucatán. Estos observatorios cuentan con investigadores con experiencia en trabajos colectivos de este tipo como lo fue el análisis de la campaña electoral federal del 2012 en México o el proyecto de Género convocado por Global Media Monitory Proyect en el 2015, de ahí que este ejercicio de análisis permitió establecer una agenda unificada, en cuanto al conocimiento del enfoque teórico, la metodología y la técnica seleccionada. Ahora bien, en la selección de los diarios por analizar, se estableció que cada observatorio acordara aquellos que fueran más importantes en cada entidad o ciudad de residencia del observatorio, de tal forma que fueron 12 periódicos de circulación reconocida los determinados para el análisis. Durante este proceso, el Observa/Frontera capacitó a la analista ${ }^{1}$ que participó en el registro de la información y durante septiembre, octubre y noviembre del

\footnotetext{
${ }^{1}$ Un agradecimiento especial a la estudiante C. Thania Barrios de la carrera de Ciencias de la Comunicación de la Facultad de Ciencias Humanas de la UABC, por su interés, empeño y dedicación en la tarea de analizar el diario seleccionado y documentar los datos de esta investigación.
} 
2014 se revisó una semana de cada uno de estos meses y adicionalmente, se determinó una semana aleatoria durante este periodo.

Al final del ejercicio de registro de la información, Observa/Frontera, en el análisis que realizó del diario La Crónica de Baja California (www.lacronica.com), documentó las 34 notas referidas a la problemática de medio ambiente y sus diferentes temáticas. La mayoría de ellas reseñan sucesos que relatan catástrofes provocadas por la naturaleza o el hombre, y escasamente se promueve, ya sea por el gobierno, las organizaciones de la sociedad civil $u$ instituciones educativas, programas, acciones o campañas para la preservación del medio ambiente.

Asimismo, fue notorio que el periodismo, por lo menos de este diario, no está capacitado y tal vez, ni siquiera interesado en la complejidad que implica el medio ambiente, a pesar de que la ciudad de Mexicali, capital del estado de Baja California, donde se ubica este periódico, es una zona desértica, con problemas de contaminación de diferente tipo y con frecuencia con temperaturas de 50 grados Celsius en la temporada de verano, que en conjunto hacen de la región, para quienes la habitan, un problema ambiental de cuidado extremo, ya sea por aspectos de la conservación del clima, el consumo de agua o de energía eléctrica. De ahí el interés de Observa/Frontera por participar en el proyecto de analizar en un diario bajacaliforniano los temas sobre el medio ambiente y sus principales problemáticas.

\section{OBJETO DE ESTUDIO Y OBJETIVOS DE LA INVESTIGACIÓN}

Como se refirió en párrafos anteriores, el Observatorio Veracruzano de Medios (ObVio) convocó a los observatorios miembros de la ROM-CONEICC a participar de una investigación colectiva para estudiar las representaciones mediáticas en la prensa mexicana, y determinó como objeto de estudio el tema del medio ambiente y sus principales problemáticas (Observatorio Veracruzano de Medios, 2014).

Al respecto, definió como objetivos de la investigación los siguientes, mismos que se adecuaron a las características de los diarios analizados por cada observatorio:

- Conocer la cantidad, frecuencia y ubicación de la información que la prensa publica sobre los temas medioambientales.

- Conocer los temas que los periódicos analizados le dedican ubicación relevante, y

- Conocer los actores, dependencias y organismos sociales que aparecen en las notas informativas (o ausencias) y algunos argumentos. 
Para cada uno de estos objetivos ObVio (2014) estableció un conjunto de preguntas de investigación que determinaron los criterios de análisis de contenido, las cuales fueron las siguientes:

- ¿Cuál es el grado de atención que concede la prensa impresa a los temas de medio ambiente?

- ¿Cuáles fueron los temas y subtemas que sobre asuntos relacionados con el medio ambiente recibieron mayor atención por parte de la prensa?

- ¿Qué actores escenifican este escenario?

Por otra parte, Observa/Frontera, dentro de su planteamiento metodológico tiene como hipótesis de trabajo, la siguiente expresión:

Los diarios de Baja California ${ }^{2}$, en los temas sociopolíticos, ambientales, culturales, educativos y de salud reproducen las agendas nacionales y los fenómenos políticos locales, y se asumen como portavoces de los intereses de los grupos de poder en los ámbitos económicos y/o políticos (Observa/Frontera, 2010).

\section{ABORDAJE TEÓRICO}

Es innegable que los MCM son, en gran parte, constructores de la realidad social contemporánea por lo que hacer un análisis y reflexionar sobre cómo se plasma la problemática en la frontera norte de México en la producción mediática de temáticas sociales, en este caso de medio ambiente, lo cual representa una oportunidad para estudiar sus manifestaciones y evolución en la sociedad fronteriza.

Abordar la condición mediática de los procesos periodísticos posibilita su explicación a partir de varias teorías, una de ellas es la Agenda Setting desarrollada por McCombs y Shaw (1972) y continuada por Rogers y Martin (1988), Shaw y Martin (1992) y Scheufle (2000), entre otros. El postulado central de la Agenda Setting es que "los medios de comunicación masivos establecen la agenda en cada campaña política, conformando las actitudes mayoritarias respecto de los problemas políticos" (McCombs y Shaw, 1972: 177), de ahí que es notorio que los medios, ya sean electrónicos o impresos, a partir de las temáticas y de los propios actores que se visibilizan en el espacio público, asumen sus posiciones acorde a las perspectivas de los aconteceres que promueven.

Por otra parte, las agendas de los medios pueden componerse de un conjunto acciones igual de amplio que la propia actividad social, política o económica puesta en marcha durante eventos o aconteceres significativos. Sin embargo, es notorio que el

\footnotetext{
2 Para este ejercicio el diario utilizado fue La Crónica de Baja California.
} 
énfasis de los estudios relacionados con la Agenda Setting, demuestran la preferencia de los medios por destacar los grandes temas públicos. La evidencia que demuestra tal acentuación es que en la medida en que dichos temas sean lo suficientemente notorios mediáticamente, existe la posibilidad de que los mismos sean priorizados por las audiencias (McCombs y Bell, 1996 y McCombs y Reynolds, 2002). Igual subrayado ocurre con los propios actores o fuentes informativas, cuyos valores que representan suelen ser sobredimensionados con la finalidad de destacar sus virtudes o defectos, según sea el caso. Otro rasgo significativo de la teoría de la Agenda Setting, ocurre cuando los medios al presentar un objeto suelen acentuar, ya sea positiva o negativamente, los atributos del mismo. Para los efectos de la visibilidad mediática (Thompson, 1997), en la promoción de las campañas ambientales, es notoria la forma en que se destacan los atributos de las acciones gubernamentales o de las fuentes que declaran sobre el tema, pero también de denostación de aquellas acciones que omiten, ocultan o tergiversan información sobre los accidentes causados por grupos de poder.

En estos dos ámbitos, de los más representativos de la teoría de la Agenda Setting, se evidencian los dos niveles en que opera la misma. El primer nivel, acontece mediante la prominencia del objeto (actor o institución). El segundo nivel es la exaltación del atributo de dicho objeto (Shaw y McCombs, 1977). Este último nivel de la Agenda Setting, se le ha vinculado fuertemente con otro concepto teórico del análisis de los medios, se trata del framing o encuadre, propuesto por McCombs y Evatt (1995), Scheufele (1999) y Reese (2001). Ahora bien, la técnica utilizada para el trabajo de relevamiento de la información es el análisis de contenido propuesto por autores como Berelson (1952) permite analizar el contenido manifiesto de la comunicación a partir de describir sistemática, objetiva y cualitativa, la información que se desee registrar. De ahí que esta técnica, es eficaz para analizar trozos de contenido de información y compararlos con otros o con fuentes diversas de información, por ello es que el análisis de contenido está centrado en el qué de la información.

El Observatorio Global Mediático de la Frontera Norte, utiliza esta técnica en sus trabajos de análisis y en particular se centra en el modelo de Laurence Bardin (1977) que se describe a continuación: 
Gráfico 1. Modelo de análisis de contenido

Desarrollo de un modelo análisis de contenido

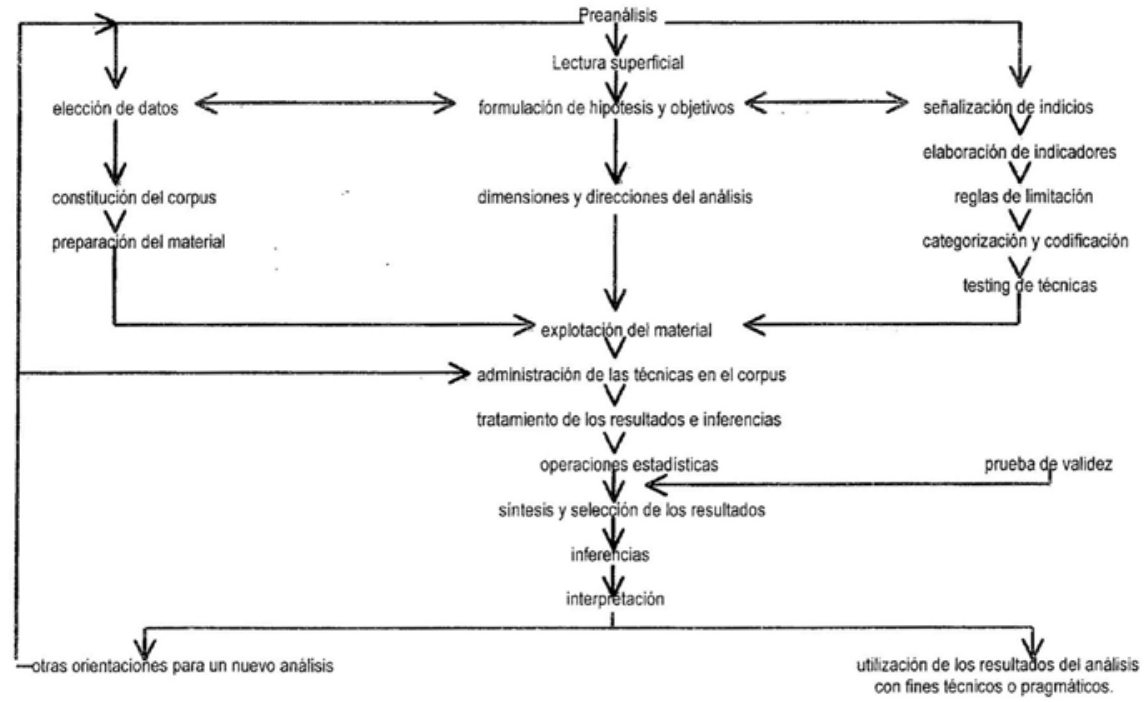

Para los efectos del análisis que se propone, el nivel de estudio fue abordado a partir de las categorías más significativas como los temas más sobresalientes junto con otros componentes como el género periodístico preferentemente utilizado, la autoría de la redacción de la nota y la valoración asignada para la misma, así como la frecuencia de las notas en cada diario. No se logró realizar un análisis estadístico más amplio por lo escaso de las notas que fueron registradas durante el tiempo indicado por la muestra establecida.

\section{CONTEXTO PERIODÍSTICO EN BAJA CALIFORNIA}

En Baja California hay 24 medios impresos. Los más antiguos e importantes son La Voz de la Frontera y El Mexicano y los más contemporáneos son La Crónica, Frontera y Vigía (Ortiz Marín, coord., 2006). En este trabajo se utilizaron las notas de La Crónica de Baja California, en el tema seleccionado de medio ambiente expresado en diferentes ítems para su registro como fenómenos naturales, desastres, cambio climático, entre otros. A continuación se documentan algunos de los rasgos más importantes de este periódico.

La Crónica de Baja California (Mexicali, Baja California) surgió el 7 de noviembre de 1990, en un principio con un formato tipo periódico. Diario que además de la forma impresa de tipo estándar como lo hacen la mayoría de los periódicos en la actualidad, 
también agrega el formato on line. La presentación del diario, en cuanto a diseño, no cambia de un modelo a otro; sin embargo, en cuanto ha contenido, la página de internet de La Crónica ofrece espacios adicionales para información diversa, la cual está compuesta por las secciones: general, deportes, sociales, espectáculos y clasificados. En la sección general se cuenta con espacios como: Mexicali, nacional, internacional, San Luis, editorial, policíaca y regional, y ocasionalmente se dedica una página a Especiales, una sección con información de acontecimientos relevantes del momento. La Crónica publica en promedio 48 páginas diarias, 30 para información general, seis para deportes y de 10 a 12 para sociales y entretenimiento, con un tiraje diario de 25 mil ejemplares y con un costo de cinco pesos (Asociación de Editores de los Estados, 2014). Su actual presidente es Juan F. Healy, Loera y el director general es Luis Alejandro Bernal García, quien también funge como director de Frontera de Tijuana, Frontera de Ensenada y el Imparcial de Sonora. El director para Baja California es Ricardo Salido Gaxiola. Estos conjuntos de diarios junto con otros tres en otras entidades federativas, forman parte de la cadena Periódicos Healy y ésta última es miembro de la Sociedad Interamericana de Prensa (SIP) (www.sipiapa.org).

\section{METODOLOGÍA Y ORGANIZACIÓN DE LA INVESTIGACIÓN}

La metodología utilizada para este ejercicio de análisis estableció como objeto central, reconocer la importancia del estudio del tema: medio ambiente; para ello se reconoce que los MCM no sólo reflejan el acontecer sino que plasman y construyen realidad referente a la problemática en cuestión y es a través del análisis de la prensa, para el caso el diario: La Crónica de Baja California, como se procedió a realizar el estudio de las representaciones mediáticas que este periódico produce en su cotidianeidad informativa.

En el proceso de análisis se diseñó un protocolo de investigación ex profeso; en una primera instancia, se elaboró un marco referencial que dio cuenta de las principales características de este periódico fronterizo de Baja California, seleccionado para el análisis de contenido periodístico. En un segundo momento, se aplicó una metodología con un enfoque cuantitativo para el análisis de contenido de las notas del diario elegido y relacionada con la temática seleccionada (Krippendorff, 1990).

- Corpus del análisis. Para el caso, el método de análisis se sustentó en los siguientes parámetros: a) Utilización del método de análisis de contenido propuesto por autores como Berelson (1952), Bardin (1977), Krippendorff (1990), como se indicó anteriormente. En lo particular se optó por la propuesta de Laurence Bardin como modelo para llevar a cabo el análisis del contenido de las notas periodísticas; b) Determinación de una muestra aleatoria para el análisis del diario seleccionado; c) Capacitación de un estudiante acerca de la téc- 
nica de análisis de contenido y e) Elaboración de diferentes instrumentos y apoyos tecnológicos para la captura y codificación de la información periodística.

- Instrumentos de análisis. Se diseñaron y aplicaron varios instrumentos para el análisis de la información periodística como fueron: Manual de análisis propuesto por ObVio (2014); una plantilla de codificación; captura de notas realizada mediante la consulta de las páginas on line del diario seleccionado en los días preestablecidos y su posterior registro en el programa Laserfiche para integrar la información.

Algunos de los criterios de registro fueron: a) se consideró como objeto del análisis la nota periodística; b) se analizó únicamente el género informativo (con lo cual se excluyeron editoriales, columnas, caricaturas y reportajes) y c) se codificaron las fuentes que cada información utilizaba como declarantes. También se identificaron otros rasgos de la información periodística codificada como: su ubicación dentro del diario (portada o interiores), procedencia de la nota, lugar de origen de la misma, título de la nota y la posible referencia al cambio climático.

- Muestra utilizada y cantidad de notas analizadas. El Observatorio Veracruzano de Medios, determinó la muestra utilizada, la cual consistió en cuatro semanas, tres naturales y una aleatoria a partir de septiembre, las cuales fueron las siguientes:

Tabla 1. Muestra estadística

\begin{tabular}{|l|l|l|}
\hline \multicolumn{3}{|c|}{ FECHAS DE ANÁLISIS 2014} \\
\hline Semana natural 1 & 15 al 21 de septiembre \\
\hline Semana natural 2 & 20 al 26 de octubre \\
\hline Semana natural 3 & 24 al 30 de noviembre \\
\hline Semana aleatoria & DÍAS BASE & $\begin{array}{l}\text { DÍAS ALTERNATIVOS POR SI NO } \\
\text { SE PUBLICA EL PERIÓDICO } \\
\text { Lunes- 13 Octubre } \\
\text { Martes- 04 noviembre } \\
\text { Lunes- 03 noviembre } \\
\text { Martes- 02 septiembre } \\
\text { Miércoles- 15 octubre } \\
\text { Jueves- 09 octubre } \\
\text { Viernes- 26 septiembre } \\
\text { Sábado- 22 noviembre } \\
\text { Domingo- 09 noviembre } \\
\text { Jueves- 25 septiembre } \\
\text { Viernes- 14 noviembre } \\
\text { Sábado- 15 noviembre } \\
\text { Domingo- 19 octubre }\end{array}$ \\
\hline
\end{tabular}


Ahora bien, el diario La Crónica de Baja California, sólo publicó en este periodo de la muestra, la cantidad de 34 notas informativas que se clasificaron dentro del rubro temas del medio ambiente; sin embargo, la suma total de los seis observatorio es de 1.751 notas. Total al cual lo aportado por el periódico bajacaliforniano representa el $1,94 \%$ del total de notas periodísticas registradas en el proyecto. Aun así resulta significativo el análisis, pues como lo indica Berelson (1952) también las ausencias son importantes de estudiar.

Tabla 2. Distribución de las notas en la muestra

\begin{tabular}{|l|l|c|}
\hline \multicolumn{1}{|c|}{ SEMANA } & \multicolumn{1}{c|}{ FECHA } & CANTIDAD \\
\hline 1‥ Semana natural & 15-21 septiembre & 30 \\
\hline 2a. Semana natural & 20-24 octubre & 3 \\
\hline 3a. Semana natural & 24-30 noviembre & 0 \\
\hline Semana aleatoria & Sept-oct-nov. & 1 \\
\hline
\end{tabular}

A continuación se procede a la descripción y análisis de los principales resultados a partir de la exposición de los datos, integrados en la base de datos nacional generada por la información proporcionada por los seis observatorios participantes en la investigación; información que a su vez se contrasta con los datos derivados del análisis de las notas del diario de Baja California.

\section{ANÁLISIS DE LOS RESULTADOS}

Uno de los aspectos importantes de la teoría de la Agenda Setting que refieren McCombs y Shaw (1972) es el establecimiento de la agenda; es decir, son aquellos temas a los cuales los medios de comunicación dan mayor difusión y preferencia en sus espacios informativos acentuando su posicionamiento $y$, por ende, ocurre la posibilidad de que los mismos sean priorizados por las audiencias (McCombs y Bell, 1996; McCombs y Reynolds, 2002). 
Tabla 3. Temas por La Crónica de Baja California

\begin{tabular}{|l|c|}
\hline \multicolumn{1}{|c|}{ TEMA } & CANTIDAD \\
\hline 1. Fenómenos naturales & 24 \\
\hline 2. Desastres industriales & 1 \\
\hline 5. Deforestación y/o daños en cultivos agrícolas & 5 \\
\hline 6. Cambio climático & 1 \\
\hline 10. Desastres humanos & 1 \\
\hline 11. Políticas ambientales & 1 \\
\hline 12. Acciones de protección & 1 \\
\hline
\end{tabular}

Desde la perspectiva teórica y la metodología de análisis de contenido antes enunciada, el registro de los temas cobra relevancia empírica; sin embargo, para el caso de Baja California, la cantidad de noticias fue menor como ya se ha demostrado en comparación con el contexto nacional.

Al respecto, es posible identificar que la información está concentrada en uno de los temas: Fenómenos naturales, que ocupó el 70 por ciento, con 24 de las 34 notas. La razón fue que en la semana del 15 al 21 de septiembre ocurrió el arribo del huracán Odile a las costas de la península bajacaliforniana, concretamente en Baja California Sur, cuyos efectos impactaron también a diversas zonas del estado de Baja California.

El otro aspecto ambiental que ocupó cinco notas de las 34, fue lo relacionado con deforestación y/o daños a cultivos agrícolas. En este caso, las notas se centran en destacar los graves incendios forestales que ocurrieron tanto en Baja California como en el estado de California, EUA, que devastaron cientos de hectáreas que llegaron a sumar en el año hasta 100 incendios en el 2014 (Molina, 2014).

Ahora bien, en la base de datos nacional los temas que mayor porcentaje ocuparon también fueron los de Fenómenos Naturales (que implican la presencia de huracanes o tormentas tropicales) con el 43,9 por ciento y las lluvias intensas con el 16 por ciento. Siendo coincidente en este mismo renglón con lo registrado por el diario bajacaliforniano de La Crónica.

En cuanto a la ubicación de las 34 notas en las páginas de La Crónica de Baja California, todas fueron en interiores, lo cual confirma que la atención al tema del medio ambiente, no es un tema de la agenda del diario. 
Tabla 4. Ubicación de la nota

\begin{tabular}{|l|c|c|}
\hline Portada superior & 78 & $4 \%$ \\
\hline Portada inferior & 71 & $4 \%$ \\
\hline Interiores & 1,602 & $92 \%$ \\
\hline Total & 1,751 & 100 \\
\hline
\end{tabular}

Este dato no difiere de la tendencia en el total de las notas de la base nacional pues el $92 \%$ de las 1.751 se ubican en páginas interiores, según lo indica la tabla 4 . Sin embargo, en el caso del diario bajacaliforniano, fue notorio que la distribución por semanas de las notas si tiene una fuerte concentración en la primera semana de septiembre como lo indica la tabla 2. Distribución de las notas en la muestra, que se mostró en párrafos anteriores.

Otro de los aspectos significativos del análisis del contenido es que permite identificar las diferencias destacadas en el tipo de fuentes que se utilizan en el trabajo periodístico, merced a la confiabilidad y también al prestigio de quien declara. Una de las razones por la cual se puede explicar este proceder, es que los reporteros se desempeñan en una organización diseñada para dar cuenta, a una gran velocidad, de tipificaciones de acontecimientos (no sucesos espontáneos) lo cual depende de la estabilidad de fuentes que les provean de información pre-elaborada y adecuada a su ritmo de producción (Tuchman, 1983).

Tabla 5. Fuentes declarantes de La Crónica de Baja California

\begin{tabular}{|l|c|}
\hline \multicolumn{1}{|c|}{ FUENTES } & FRECUENCIA \\
\hline Dependencia o funcionario del gobierno estatal & 20 \\
\hline Dependencia o funcionario del gobierno federal & 11 \\
\hline Gobernador del estado & 1 \\
\hline Organización de la Sociedad Civil internacional & 1 \\
\hline Colonos, vecinos, habitantes de poblados & 1 \\
\hline Total & 34 \\
\hline
\end{tabular}

Respecto de la anterior tabla se aprecia que en el caso del diario La Crónica, éste da preferencia a las autoridades gubernamentales, ya sea del nivel estatal o del gobierno federal en el tema de los problemas de medio ambiente, por lo que escasa- 
mente la ciudadanía tiene una voz en este tópico, ya sea mediante la opinión de las organizaciones no gubernamentales o los directamente implicados en la problemática ambiental.

\section{CONCLUSIONES}

El análisis de las noticias relacionadas con el medio ambiente publicadas por La Crónica de Baja California confirma que la atención a las diferentes expresiones de la condición ambiental ocurre principalmente cuando los hechos aluden a sucesos catastróficos de manera preminente; aunado a lo anterior, también los registros del conjunto de diarios analizados por los seis observatorios mediáticos que participaron en el estudio, muestra en algunos casos similitudes en el tratamiento informativo.

Por tal razón de la información derivada de las representaciones mediáticas visibilizadas por el diario La Crónica de Baja California y en respuesta a las interrogantes planteadas como ejes de la investigación, el análisis da cuenta en cada una de ellas de lo siguiente:

\section{¿Cuál es el grado de atención que concede la prensa impresa a los temas de medio ambiente?}

La Crónica de Baja California, le concedió atención a los sucesos de medio ambiente en la medida en que estos fueron significativos para la representación mediática por el tipo de evento que ocurrió, lo cual se demuestra con el 88 por ciento de las notas publicadas por dicho diario con respecto al acontecer que se ubican en la primera semana de la muestra seleccionada que corresponde entre el 15 al 21 de septiembre, periodo en el cual de las 34 notas, 30 de ellas abordan el suceso del impacto del huracán Odile y su efectos en los daños ocasionados a la población de Baja California Sur, pero también a los ecosistemas.

Esto es coincidente con el estudio de Shanahan (2007), que a la ocurrencia de noticias sobre desastres climáticos que conmueven por su impacto en la estabilidad o los destrozos ocasionados por la furia de los elementos, los medios destacan estos hechos noticiosos, lo cual también viene a demostrar que el periodismo ambiental que practica el periódico estudiado se inscribe en el modelo empírico que aborda la conflictiva ambiental sin la preparación adecuada (Gavirati, 2007).

Aunado a lo anterior, todas las notas publicadas por el diario La Crónica, fueron en páginas interiores, lo cual no difiere de las notas recabadas por el conjunto de diarios analizados en el proyecto, donde un 92 por ciento de las notas publicadas (1.602) es en páginas interiores de los diarios de las 1.751 notas analizadas y registradas por los 
seis observatorios que participaron del proyecto. Este hecho indica la gravedad de la falta de conciencia ambiental que se tiene en ciertos sectores del periodismo mexicano.

\section{¿Cuáles fueron los temas y subtemas que sobre asuntos relacionados con el medio ambiente recibieron mayor atención por parte de la prensa?}

En este rubro, los principales temas y subtemas abordados por La Crónica de Baja California fueron los sucesos referidos a los fenómenos naturales con un 70 por ciento como fue el impacto del huracán Odile a las costas de la península bajacaliforniana, concretamente en Baja California Sur, cuyos efectos afectaron diversas zonas del estado de Baja California.

Este dato también es similar a los registros nacionales que indican que la publicación de las noticias referentes a huracanes o ciclones ocuparon el 43,9 por ciento y el 16 por ciento fue para lluvias intensas (un 59,9 por ciento acumulado) en las diferentes entidades en que se ubican los periódicos analizados por los observatorios partícipes del estudio.

Ahora, bien hay sólo tres noticias que refieren la difusión de otro tipo de acciones en torno al medio ambiente. Una que atiende la categoría de cambio climático (relacionada con los efectos de esta condición en la escases de lluvia y en la desaparición de cierto tipo de fauna); otra que registra el tema de políticas ambientales (alude a acciones del gobierno estatal de Baja California en una campaña de acopio de pilas para uso de aparatos electrónicos) y una tercera que da cuenta de la contaminación del aire (en la cual se alerta a la población por la pésima calidad del aire).

\section{¿Qué actores escenifican este escenario?}

Los actores o en este caso los sujetos declarantes que participan como fuentes de información son esenciales en un ejercicio periodístico centrado en la declaración de sujetos con credibilidad para aportar información sobre el tema en cuestión, tal como lo demuestran los datos de la tabla 5 "Fuentes declarantes de La Crónica", que registra un 94 por ciento de autoridades gubernamentales y apenas un 6 por ciento de la sociedad civil ya sean en la voz de las organizaciones no gubernamentales o ciudadanos en lo particular.

Son las fuentes que tienen la condición de autoridad a las que se suele citar de manera privilegiada y reiterada. Como señalan McCombs y Weaver (1973), estas fuentes legitimadas y legitimables sirven de orientación para que los públicos conformen un mapa del contexto a partir de las experiencias personales y en función de 
ello, puedan ajustar su actuar cotidiano. Esto es importante al tratarse de sucesos que refieren desastres, seísmos o fenómenos cuya naturaleza implica el riesgo de vida, ya que los directamente implicados demandan información autorizada para ajustar su actuar a las condiciones prevalecientes.

Para finalizar este apartado, si la teoría de Agenda Setting se sitúa en el espectro de focalizar las temáticas para que el público tenga en qué pensar, el aporte de Cohen (1993) al respecto sobre el éxito que tienen los medios al decirle a los públicos sobre qué tienen que pensar. Si esto fuera así, surge justamente la contradicción pues ante la escases de información orientadora, preventiva y de toma de conciencia de la problemática del medio ambiente en sus diferentes manifestaciones, surge la hipótesis de que en el caso de los diarios estudiados, tanto a nivel local como fue $L a$ Crónica de Baja California, como en otros medios, la apuesta es a invisibilizar esta condición.

Ahora bien, en el campo de las políticas públicas, Borrego (2006) señala que hay avances en cuanto a la creación del Derecho a la información ambiental y la formación del Sistema Nacional de Información Ambiental, pero continúan las limitaciones para que el ciudadano pueda ejercer su derecho de información ambiental, como por ejemplo en auditorías ambientales, derecho de petición de información ambiental y la participación pública en lo que respecta a la posibilidad de solicitar una consulta pública acerca de una obra o proyecto que afecte el medio ambiente y la reparación del daño o indemnización en caso de un accidente ambiental, como lo ocurrido en 2014 con el derrame de 40 mil metro cúbicos de sulfato de cobre en las aguas del Río Sonora por la minera Grupo México que afectó las siembras, el agua para consumir y la vida de la flora y fauna de la región de Sonora, además de ser calificado como el peor desastre ambiental (Miranda, Larios y Alanís, 2014).

\section{BIBLIOGRAFÍA}

ASOCIACIÓN DE EDITORES DE LOS ESTADOS (2014): Tarifario 2014. México, D. F.: Asociación de Editores de los Estados. Disponible en http://www.aee.com.mx.

BARDIN, L. (1977): Análisis de contenido. Madrid: Akal.

BERELSON, B. (1952): Content Analysis in Communications Research. New York: Free Press.

BORREGO, N. (2006): "Las políticas públicas de protección al medio ambiente en Norteamérica", Revista Mexicana de Estudios Canadienses, № 11, pp. 163-192.

COHEN, B. (1993): The press and foreign policy. University of California. 
DÍAZ NOSTY, B. (2009): “Cambio climático, consenso científico y construcción mediática. Los paradigmas de la comunicación para la sostentabilidad", Revista Latina de Comunicación Social, no 64. DOI: http://dx.doi.org/10.4185/RLCS-64-2009-808-99-119.

GAVIRATI, P. (2013): "Mediatizar el ambiente; ambientizar los medios. Tensiones en torno al discurso periodístico sobre el cambio climático", en Fernández, R. (dir.) y Mancianas C. (coord.): Medios de comunicación y cambio climático. Sevilla: Fénix, pp. 217-232.

KRIPPENDORFF, K. (1990): Metodología de análisis de contenido. Teoría y práctica. Barcelona: Paidós.

MARTÍN SERRANO, M. (1977): La mediación social. Madrid: Akal.

MARTíN SERRANO, M. (1985): "La mediación de los medios de comunicación". En Moragas, Miquel de (ed.): Sociología de la comunicación de masas. I. Escuelas y autores. Barcelona: Gustavo Gili, pp. 141-162.

McCOMBS, M. E. y BELL, T. (1996): "The agenda-setting role of mass communication", en Salwen, M. y Stacks. D. (eds.): An integrated approach to communication theory and research. Mahwah, NJ: Lawrence Erlbaum, pp. 93-110.

McCOMBS, M. E. y EVATT, D. (1995): "Issues and attributes: exploring a new dimension in agenda setting", Comunicación y Sociedad, vol. 8, nำ1, pp. 7-32.

McCOMBS, M. E. y REYNOLDS, A. (2002): "News influence on our pictures of the world", en Bryant, J. y Zillmann, D. (eds.): Media effects. Advances in theory and research. Mahwah, NJ: Lawrence Erlbaum Associates, pp. 1-18.

McCOMBS, M. E. y SHAW, D. L. (1972): "The agenda-setting function of mass media", Public Opinion Quarterly, vol. 36, no 2, pp. 176-187. DOI: http://dx.doi.org/10.1086/267990.

McCOMBS, M. E. y WEAVER, D. (1973): “Voters' need for orientation and use of mass communication", Annual Meeting of the International Communication, Montreal, Canadá, 25-28 de abril de 1973.

MIRANDA, F., LARIOS, F. y ALANÍS, R. (2014): “En Sonora, "peor desastre» ambiental minero: Guerra”, Milenio. Disponible en http://goo.gl/LHiCVV. Consultado el 27 de agosto de 2015.

MOLINA, O. (2014): "100 incendios forestales van en el 2014", La Crónica, p. 13.

OBSERVA/FRONTERA (2010): “Observatorio Global Mediático de la Frontera Norte de la Universidad Autónoma de Baja California. Documento ejecutivo”, Observa/Frontera. Disponible en http://observafronterauabc.blogspot.mx.

OBSERVATORIO VERACRUZANO DE MEDIOS (2014): “Esto es ObVio", Observatorio Veracruzano de Medios. Disponible en www.uv.mx/blogs/obvio.

ORTIZ MARÍN, Ángel Manuel (coord.) (2006): Los medios de comunicación en Baja California. México: Porrúa / Universidad Autónoma de Baja California. 
REESE, S. D., GANDY, O. H. y GRANT, A. E. (2001): Framing public life. Perspectives on media and our understanding of the social world. Mahwah, NJ: Lawrence Erlbaum Associates.

ROGERS, E. M. y MARTIN, S. E. (1988): "Agenda setting research: where has it been? Where is it going?", en Anderson, J. A. (ed.): Communication Yearbook, Vol. 11. California: Sage, pp. 555-594.

SCHEUFLE, D. (1999): "Framing as a theory of media effects", Journal of Communication, vol. 49, no 1, pp. 103-122. DOI: http://dx.doi.org/10.1111/j.1460-2466.1999.tb02784.x.

SCHEUFLE, D. (2000): “Agenda-setting, priming and framing revisited: another look at cognitive effects of political communication", Mass Communication and Society, vol. 3, no 2-3, pp. 297-316. DOI: http://dx.doi.org/10.1207/S15327825MCS0323_07.

SHANAHAN, M. (2007): "Hablar de una revolución: el cambio climático y los medios de comunicación", International Institute for Environment and Development. Disponible en http://pubs.iied.org/pdfs/17029SIIED.pdf. Consultado el 2 agosto de 2015.

SHAW, D.L. y McCOMBS, M. (1977): The emergence of American political issues. St. Paul: West.

SHAW, D.L. y MARTIN, S.E. (1992): "The function of mass media agenda setting", Journalism Quarterly, vol. 69, no 4, pp. 902-920. DOI: http://dx.doi.org/10.1177/107769909206900410.

THOMPSON, J. (1997): Los medios y la modernidad. Una teoría de los medios de comunicación. Barcelona: Paidós.

TUCHMAN, G. A. (1983): La producción de la noticia. Estudio sobre la construcción de la realidad. Barcelona: Gustavo Gili.

UNESCO (2015): "Mensaje de la Directora General de la UNESCO, Irina Bokova, con motivo del Día Mundial de Lucha contra la Desertificación", UNESCO. Disponible en http://goo.gl/b57fLJ.

YONA, L. (2015): “El Ártico continúa en peligro tras la cumbre de Anchorage", Periodistas en Español. Disponible en http://goo.gl/2jJ5s5. Consultado el 3 de septiembre de 2015.

Ángel Manuel ORTIZ MARín es Doctor en Comunicación Social por la Universidad de La Habana. Profesor-investigador de tiempo completo en la Facultad de Ciencias Humanas de la Universidad Autónoma de Baja California (UABC), cultiva la línea de investigación de las representaciones mediáticas en los medios de frontera. Actualmente coordina el Observatorio Global Mediático de la Frontera Norte de la UABC. Correo electrónico: mortiz@uabc.edu.mx. 